Bull. Mater. Sci., Vol. 6, No. 1, February 1984, pp. 53-57.

(C) Printed in India.

\title{
Electron transport in non-crystalline garnet films
}

\author{
D BAHADUR, D ROY, B SINGH* and D SARAN* \\ Advanced Centre for Materials Science, Indian Institute of Technology, Kanpur 208016 , \\ India \\ * Department of Physics, Indian Institute of Technology, Kanpur 208 016, India
}

\begin{abstract}
Thin films of yttrium iron garnet (YIG) and Gd-substituted YIG of different thickness have been prepared by flash evaporation. The surface DC and AC electrical resistivity and thermopower in these films have been studied. The results are explained on the basis of Mott and Davis model in which narrow tails of localised states exist at the extreme valence and conduction bands and a band of localised levels near the middle of the gap. For the temperature range studied, the main conduction mechanism is on account of excitation of carriers into localised states at the band edges and hopping at energies close to the band tails.
\end{abstract}

Keywords. Thin films; non-crystalline garnet; electron transport; activation energy.

\section{Introduction}

The electron transport on polycrystalline and single crystals of yttrium iron garnet and its derivatives has been studied and understood in the last couple of decades (Elwell and Dixon 1968; Larsen and Metselaar 1976; Bahadur et al 1981). Some attempts have also been made to prepare non-crystalline garnets and characterise them (Popma and Van Diepen 1974; Cuomo et al 1972). We have recently prepared non-crystalline garnet by flash evaporation and investigated the magnetic domains and structural transformations (Bahadur and Rai 1980). To get further insight into the electronic behaviour of these films, a number of films of YIG and $Y_{2}$ GdIG have now been prepared and its electron transport behaviour has been studied. The electron transport in bulk samples has been studied recently (Bahadur et al 1981). It was therefore considered worthwhile to investigate the effect of disorder and film thickness on the electron transport studies on the films of the same compositions.

\section{Experimental}

The polycrystalline samples of YIG and $\mathrm{Y}_{2}$ GdIG were prepared by the usual ceramic method of mixing and sintering of constituent oxides at $1350^{\circ} \mathrm{C}$. These samples were characterised by $x$-ray diffraction for a single phase. All the oxides were of high purity. Thin films of these garnets were deposited on a clean glass and quartz substrate by flash evaporation in an ultimate vacuum of $2 \times 10^{-5}$ torr. Gold electrodes were deposited on the film for electron transport studies. Thickness of these films was measured. The standard error in thickness measurement is $\pm 20 \AA$. Before the electron transport measurements, the films were annealed at $450 \mathrm{~K}$ for about $72 \mathrm{hr}$. The surface electrical resistivity (AC and $\mathrm{DC}$ ) was measured using a Keithley electrometer and $1610 \mathrm{GR}$ impedance bridge with RC oscillator and null detector. I-V characteristics were tested at intermittent temperatures to check its linearity. Thermo-eme was measured using Leeds and Northrup potentiometer. 


\section{Results and Discussion}

All the films are amorphous in nature as evident from x-ray and electron diffraction. The thickness of different YIG films were 230, 560 and $1330 \AA$. A typical plot of the surface electrical resistivity (DC) versus inverse of temperature for YIG film of $230 \AA$ is given in figure 1. Table 1 gives the activation energies of conduction for different ranges for YIG films. Resistivity data was taken while decreasing the temperature and the activation energy was seen to be lower. This could be due to the increase in grain size after crystallisation reducing the discontinuity in the film which gave an additional activation energy for the film. However, no such marked change was observed for higher thickness. Resistivity also decreases as the thickness increases and this agrees with the studies on semi-conducting films (Chopra 1969). Activation energy also shows a similar trend. We have taken thermoelectric power data versus temperature for two of the YIG films of thicknesses 560 and $1300 \AA$ and the plot for the first is given in figure 2 .

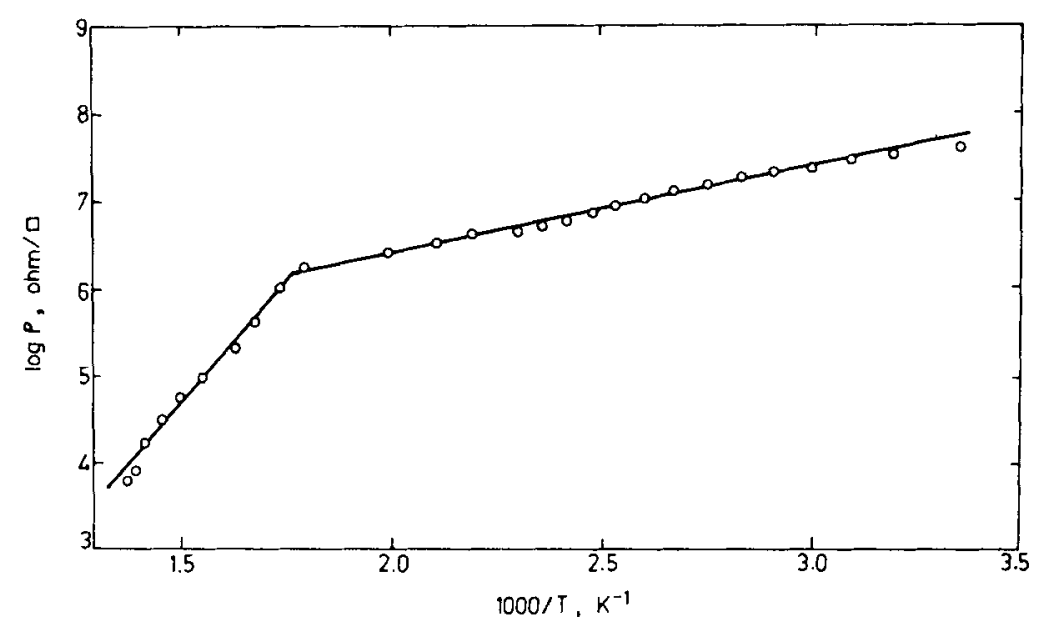

Figure 1. Plot of surface electrical resistivity (DC) versus inverse of temperature for YIG film of thickness $230 \AA$.

Table 1. Activation energies in different ranges for thin films of YIG

\begin{tabular}{ccl}
\hline $\begin{array}{c}\text { Sample's } \\
\text { thickness } \\
(\AA)\end{array}$ & $\begin{array}{c}\text { Range } \\
(\mathrm{K})\end{array}$ & $\begin{array}{c}\text { Activation } \\
\text { energies } \\
(\mathrm{eV})\end{array}$ \\
\hline 230 & $300-570$ & $0 \cdot 20$ \\
& $570-725$ & $1 \cdot 06(0 \cdot 62)^{*}$ \\
560 & $400-550$ & $0 \cdot 16$ \\
& $550-730$ & 0.80 \\
1300 & $450-540$ & 0.07 \\
\hline
\end{tabular}

*In parenthesis is given the value of activation energy while lowering the temperature 


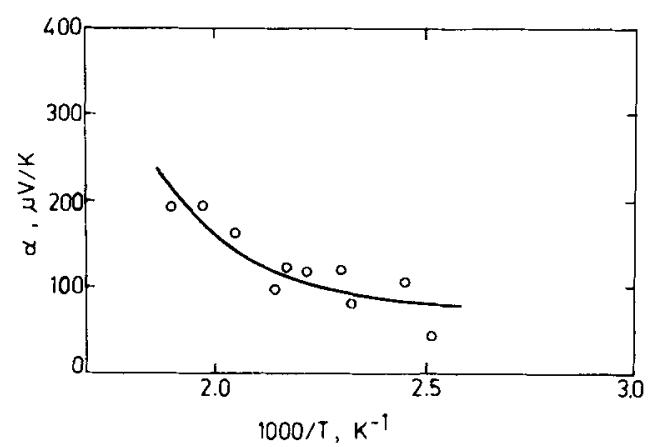

Figure 2. Plot of thermo-electric power versus inverse of temperature for YIG film of $560 \AA$ thickness.

From figure 1 and table 1, we observe two ranges for activation energies of conduction similar to that found in bulk yig earlier (Bahadur et al 1981). However, the values are less than what is observed for bulk YIG. This is to be expected if we assume band model for amorphous systems (Mott and Davis 1979; Nagels 1979) where tails of localised states and band of compensated levels within the gap could, in principle, cause lowering of activation energies.

Thermoelectric power, $\alpha$, versus inverse of temperature plot shows that $\alpha$ is positive and increases with temperature showing that the holes are the majority charge carriers. However, the values are less than the bulk values which is possibly due to higher density of states at the fermi energy.

A typical plot of surface electrical resistivity (DC) for $Y_{2}$ GdIG thin film of $420 \AA$ thickness is given in figure 3. For the same film, the log of AC resistivity is plotted against $\log$ of frequency in figure 4 . The resistivity between 290 and $490 \mathrm{~K}$ was measured for three samples of $Y_{2}$ GdIG. The activation energies of conduction and $S$ values are given in table 2. Only a small change is seen in the activation energies near $390 \mathrm{~K}$ for all the three samples. However, no such change was observed in YIG films around this temperature. This change appears to be characteristic of gadolinium substitution; the change is nearly same in all the three samples. In fact, in bulk $Y_{2} G d I G$ also, an additional range of activation energy has been observed earlier (Bahadur et al 1981).

It is interesting that the frequency dependence of resistivity varies approximately as $\omega^{s}$ where $S<1$ and lies between 0.65 and 0.9 . In figure 3(b), it is observed that the $S$ value at room temperature is 0.81 whereas at $475 \mathrm{~K}$, it is 0.78 . It has also been observed that $\rho(\omega)$ for this sample exponentially depends upon temperature.

Based on the above observations, we propose that the electronic conduction is on account of excitation of carriers into localised states at the band edges and hopping at energies close to band tails; DC resistivity in such a situation will be given by (Mott and Davis 1979).

$$
\rho=\rho_{0} \exp \left[\left(E_{a}-E_{f}+W\right) / \mathrm{kT}\right],
$$

where $E_{a}$ corresponds to the band edge, $W$ is the activation energy for hopping and $\rho_{0}$ is the pre-exponential factor which depends upon the density of states near $E_{a}$, hopping distance and the bandwidth of localised states. The essential feature of the Davis and Mott model is the narrow tails of localised states at the extremities of the valence and 


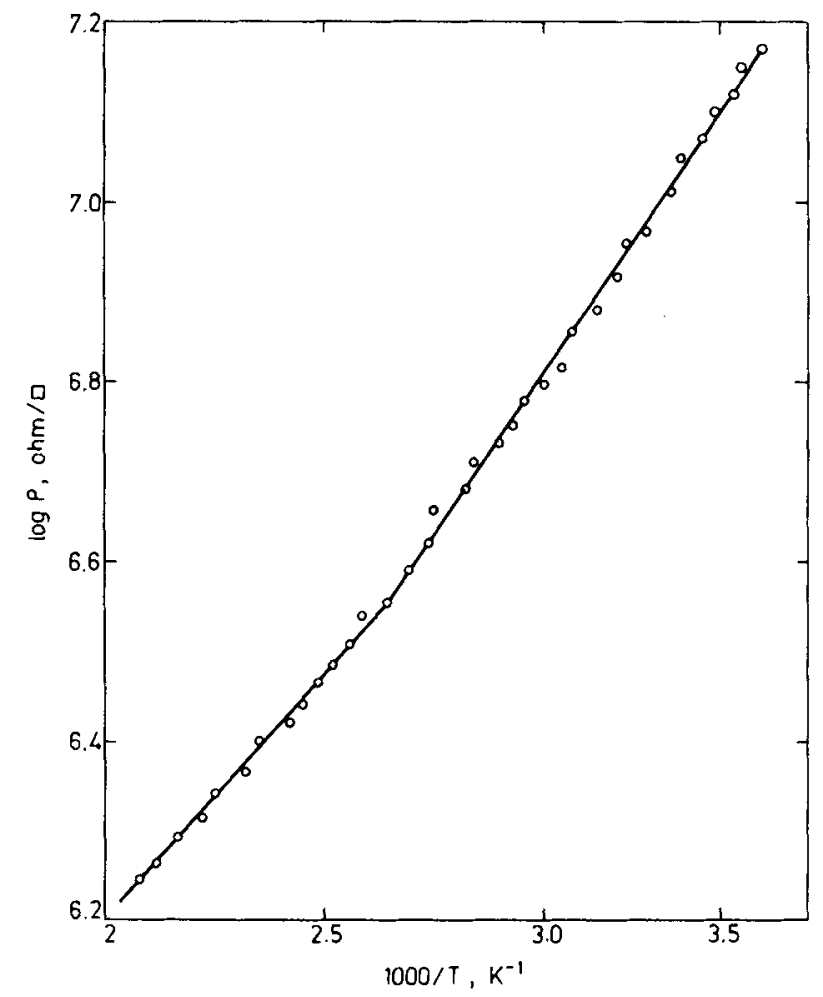

Figure 3. Plot of surface electrical resistivity (DC) versus inverse of temperature for $\mathrm{Y}_{2}$ GdIG film of $420 \AA$ thickness.

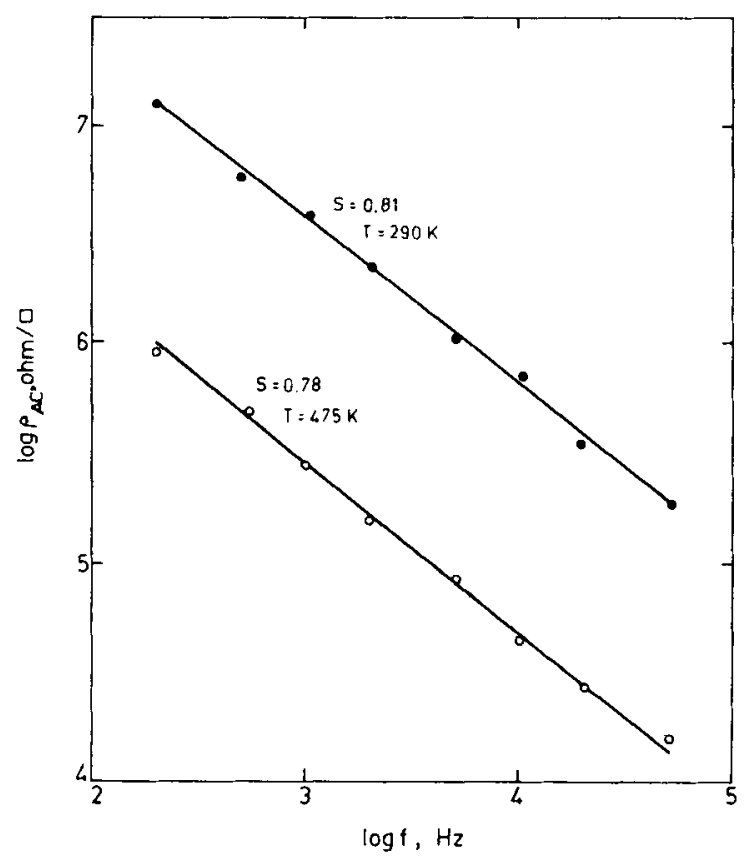

Figure 4. Plot of $A C$ resistivity versus frequency for $\mathrm{Y}_{2} \mathrm{GdIG}$ film of $420 \mathrm{~A}$ thickness. 
Table 2. Activation energies in different ranges for thin films of $\mathrm{Y}_{2} \mathrm{GdIG}$

\begin{tabular}{lccc}
\hline $\begin{array}{l}\text { Sample's } \\
\text { thickness } \\
(\AA)\end{array}$ & $\begin{array}{c}\text { Range } \\
(\mathbf{K})\end{array}$ & $\begin{array}{c}\text { Activation } \\
\text { energies } \\
(\mathrm{eV})\end{array}$ & $\begin{array}{c}\text { S value } \\
\text { at } \\
290 \mathrm{~K}\end{array}$ \\
\hline 420 & $290-380$ & $0 \cdot 14$ & 0.78 \\
& $380-475$ & 0.11 & \\
570 & $330-390$ & $0 \cdot 20$ & 0.65 \\
& $390-485$ & $0 \cdot 17$ & \\
1300 & $290-380$ & 0.16 & 0.88 \\
& $380-455$ & 0.13 & \\
\hline
\end{tabular}

conduction bands and furthermore of a band of localised levels near the middle of the gap. According to this model, three different conduction mechanisms are possible.

Extended state conduction requires a frequency-independent resistivity and the slope for $S$ and $\log \rho v s 1 / T$ be the same which are not found in the present results. However, at higher temperature, it is not ruled out. Since, most of our results are between 290 and $550 \mathrm{~K}$, our discussion would be limited for this region only. For this range of temperature it appears that the conduction in localised states at the fermienergy would not be operative. This is indicated by the exponential dependence of $\rho(\omega)$ on temperature which is characteristic of conduction in band tails. Again, it is possible that at low temperature, the conduction is through variable range hopping due to the Anderson localised states at the fermi-energy (Anderson 1958; Rao et al 1975). Detailed low temperature studies are in progress to ascertain these facts.

\section{Acknowledgement}

Thanks are due to Department of Atomic Energy for the award of research grants.

\section{References}

Anderson P W 1958 Phys. Rev. 1091492

Bahadur D and Rai K N 1980 Mater. Res. Bull. 15551

Bahadur D, Parkash O and Kumar D 1981 Bull. Mater. Sci. 3325

Chopra K L 1969 Thin film phenomena (New York and London: McGraw-Hill)

Cuomo J J, Sadagopan V, Deluco J, Chaudhary P and Rosenters R 1972 Appl. Phys. Lett. 21581

Elwell D E and Dixon A 1968 Solid State Commun. 6585

Larsen P K and Metselaar R 1976 Phys. Rev. B14 2520

Mott N F and Davis E A 1979 Electronic processes in non-crystalline materials (Oxford: Clarendon) p 199 Nagels P 1979 in Amorphous semi-conductors (ed.) M H Brodsky (Berlin, Heidelberg and New York: Springer Verlag) p 113

Popma J A Th and Van Diepen A M 1974 Mater. Res. Bull. 91119

Rao C N R, Bhide V G and Mott N F 1975 Philos. Mag. 321277 (and references therein) 